\title{
COnCEPT \\ Developing Intelligent Information Systems To Support Colloborative Working Across Design Teams
}

\author{
Aggelos Liapis ${ }^{1}$, Julia Kantorovitch ${ }^{2}$, Julian Malins ${ }^{3}$, Anastasios Zafeiropoulos ${ }^{1}$, Mieke Haesen ${ }^{4}$, \\ Marisela Gutierrez Lopez ${ }^{4}$, Mathias Funk ${ }^{5}$, Jesus Alcantara ${ }^{5}$, John-Paul Moore ${ }^{6}$ and Fiona Maciver ${ }^{3}$ \\ ${ }^{I}$ Intrasoft International, Markopoulou-Peania Avenue, Athens, Greece \\ ${ }^{2}$ VTT-Technical Research Center, Espoo, Finland \\ ${ }^{3} I D E A S$ Research Institute, RGU, Garthdee Road, Aberdeen, UK \\ ${ }^{4}$ Hasselt University-tUL-iMinds,Expertise Centre forDigital Media, Diepenbeek, Belgium \\ ${ }^{5}$ Department of Industrial Design, Eindhoven University of Technology, Den Dolech, Netherlands \\ ${ }^{6}$ Atos, Albarracín, Madrid, Spain \\ \{aggelos.liapis,anastasios.zafeiropoulos\}@intrasoft-intl.com, julia.kantorovitch@vtt.fi.,\{j.malins, f.maciver1@rgu.ac.uk\}, \\ \{mieke.haesen,marisela.gutierrezlopez@uhasselt.be\},\{M.Funk, J.munoz.alcantara\}@tue.nl\},,john-paul.moore@atos.net
}

Keywords: Business information systems, collaboration, context-awareness, architecture, design principles

Abstract: $\quad$ Rapid developments in hardware and software are creating opportunities to enhance the user experience. For example, advances in social analytics can provide near instant feedback. State of the art information extraction tools, filtering, categorization and presentation mechanisms all greatly facilitate knowledge exploitation activities. However, these technologies are not yet fully integrated into modern business systems. This paper describes research being undertaken in order to develop a new collaborative creative design platform (COnCEPT) aimed at investigating of new data-mining and collaboration technologies in order to enhance the information systems of future businesses. This paper describes the software architecture and the components, together with the design principles which underpin the design of the new COnCEPT platform, which is being developed to address the needs of professional design teams working collaboratively in a professional context.

\section{INTRODUCTION}

The work environments of the businesses of the future will place greater importance on the human dimension, making the most of workers' knowledge, skills and cultural backgrounds. This will require new types of tools, applications and services with a certain level of intelligence, including 'contextaware' tools capable of supporting working practices. These new types of context-aware tools and applications when integrated with business work-processes and software systems, will have the capacity to understand what individuals are working on, as well as the ability to support users with domain specific information.

The volume of information existing on the web is already too vast for humans to cope with, and is only predicted to continue to grow exponentially in the future. According to Gartner (2013), business data will grow by $800 \%$ in five years, with $80 \%$ of that being unstructured data. Content is growing not only in volume, but also in complexity and diversity, and this brings additional challenges. When integrated as part of a business content management platform, the advances in search, indexing and presentation technology can be used to carry out the groundwork of information processing. Domain experts such as engineers, sales people, architects and designers can undertake higher levels of knowledge work and decision-making using intelligent systems.

Future business information management tools will attempt to resemble the human ability to process knowledge by deploying various options, alternatives, views, and thinking patterns, assisting knowledge workers when making critical decisions.

Taking advantage of innovative knowledge management techniques, advances in humanmachine interaction and recent developments in 
mobile cloud communication and storage technology, new types of business applications are expected to be able to help accomplish the complexity involved in making informed decisions. This is due to their capabilities in analyzing and categorizing knowledge across any and all systems, for example, business databases, intranet, personal archives, and web sources, hence bringing what can be considered 'comprehensive' knowledge to a particular task.

The concept of context-awareness has been the subject of research for several decades. Progress has been made in theories and foundations allowing the construction of context-aware applications and context-reasoning algorithms, with the added benefit of increasing the accuracy and efficiency of algorithms for extracting high and low-level context specific data (Dey, 2001: Hong, 2009). Approaches such as activity-based computing (Aggarwal \& Human, 2011) and advances in contextual user interface development (Schmidt, 2012) provide a solid foundation to consider the user experience. However, thus far the research has focused on the context of real physical environments and mobile applications, where a variety of location-based services are being applied in leisure situations. It is evident that context-awareness is not yet part of the workplace, and is far from becoming part of existing business information systems.

A number of Web-based systems have been developed for supporting collaborative activities in the different life-cycle phases of a product's development. These include marketing, production, distribution, service, etc. Distributed product development life-cycle activities, in a globally integrated environment, are associated with the use of internet as well as with web technologies. Some product development software systems have been integrated, through web technologies, into webbased collaboration systems (Yang et al., 2003). Related to the web-based collaborative product design systems are: Immersive Discussion Tool (IDT), cPAD (Shyamsundar et al., 2002), Detailed Virtual Design System (DVDS) (Arangarasan et al., 2000), Virtual-based Collaborative Environment (VRCE) (Kan et al., 2001), Web-enabled Product Data Management (PDM) (Xu et al., 2003). Moreover, various commercial tools and open source software applications and platforms, such as the IBM- and Google-branded products, eDrawings Professional, Matrix10, and others are also available (Wiki, 2014). However, the global market still lacks in collaboration tools, focussed in serving the conceptual/creative stages and decision making techniques of the design process capable of providing both knowledge management and decision making techniques with the possibility of product design evaluation. Most collaborative tools are related more to a project- and to a PLMmanagement and less to shared creative product design environments.

In recent years, with progress in personal mobile technology, social network applications and the proliferation of choice in the smart gadget consumer market, many are wondering why their work technology experience is not as advanced as their personal home-based systems. According to the Capgemini report (2013), employees in 2014 have higher expectations of workspace technology than ever before. Today, many business content and knowledge management systems are often standalone tools with unattractive user interfaces altogether different to those used in personal settings. There is a good level of automation possible for the handling of business processes using software systems to facilitate CRM and ERP tasks. However, in most cases, they are used for reporting and statistics purposes than for example, for helping to analyze customer needs, and in designing an offer that would match customer needs in the most appropriate way, and for facilitating the individual or whilst working collaboratively with others.

There are several important issues that first need to be resolved before developing a platform which addresses the end-user's requirements. Additional research and development is required to address the following issues:

- Intelligent knowledge management tools that are able to index, categorize, and present relevant knowledge to domain experts or novices, based on their existing expertise.

- Efficient collaboration tools supporting both face-to-face and remote interactions, as well as synchronous and asynchronous interactions in contextual knowledge flows.

- Contextual awareness in the seamless linking of business knowledge and content management systems with web resources.

- Adaptable human computer interfaces that are able to provide an intuitive user experience based on familiar metaphors and concepts.

The approach adopted by the COnCEPT project seeks to address the challenges faced by businesses in the future by introducing a knowledge-centred, personalised service platform to support individual product designers when collaborating in the initial stages of ideation and product conceptualization. 
In the following section, the platform's architecture and its main building components, services, and tools are presented. The aim is to capture a common pattern, and therein to facilitate innovation in the early stages of product design. The following section describes some of the principles that underpin a platform designed to support professional design teams working in collaboration with other stakeholders. Section 3, sets out the design methodology used to approach the service design for a context-aware collaboration work process and conclusions are presented in Section 4.

\section{THE CONCEPT FRAMEWORK}

The COnCEPT platform aims to respond to a variety of use case scenarios derived from both business and educational contexts.

The design of the platform needs to closely reflect the real world to be found when designers are asked to respond to a complex design brief. The underlying principles that determine successful design solutions have to be understood in order to be able to develop appropriate software architecture and interfaces capable of supporting the design process. These principles include the need to support generative divergent thinking, which is particularly important at the early stages of the design process, followed by mechanisms that support convergent thinking and evaluation of ideas. The project has used ethnographic research methods to identify the needs of professional product designers and to identify particular opportunities for supporting the design process. Adding to the complexity of the COnCEPT platform is the need to support collaborative working, which has become more important in recent years as design teams become increasingly geographically and temporally distributed. Understanding the ways in which individuals work collaboratively, establish trust and protocols, has direct implications for the design of the software architecture and its interfaces. It is critical to understand how information is handled by designers working in a professional context. For example how designers make use of images for inspiration, to explain ideas through metaphor and analogy and when visualising concepts.

The work of product designers will be supported by several means; centrally the collection of project related information from different sources, for example, web-based and locally stored sources of information, previous experience, and associated market research. The information will be available for quick access by any member of the project team, presented visually with possibilities for rearrangement and filtering. This is intended to support collective creativity and ideation. To support the features discussed above, the high-level components of the COnCEPT framework have been defined, along with the relationships between them. This is shown in Fig. 1. The defined COnCEPT architectural components aim to support all phases of the initial design process including: 1) the initiation and management of new and existing projects, 2) the brief analysis and brainstorming, 3) the collection of relevant material in a mood-board, 4) the design of a set of concepts in a storyboard which synthesizes the existing material and tools for evaluating and selecting the most promising solution for development, 5) sketching and visualising, 6) the presentation of proposed concept(s) to clients, and 7) tools allowing the review of the development of the project.

In order to support the required functionalities in each of the above-mentioned phases, use of a Content Management System (CMS) is considered necessary as the main platform, upon which the development and interconnection of individual components can be realised. CMS modules also allow for the interlinking of the proposed tools with existing business systems. A number of the components will be based on the customization and extension of existing CMS plugins, whilst other elements will be based on the integration of external open source tools in the platform. A number of mature open source CMS platforms, such as WordPress, Drupal, and Liferay have been evaluated as possible solutions for implementing the functions envisaged for the COnCEPT platform.

To facilitate the process of knowledge exploration by domain experts, the availability of a large volume of annotated resources is essential. Annotations with well-defined semantics are required to ensure the interoperability of available information for supporting knowledge sharing and collaboration across design teams and stakeholders. Annotation mechanisms for creating or re-use of content, for example text photographs and sketches will be supported within the collaborative tools, whilst information storage and retrieval is realised based on the interconnection of components with the COnCEPT database.

Semantic technologies will be exploited to assist the accurate modelling of the information provided. The relevant ontologies and Open Linked Data (LD) vocabularies will ensure the accuracy of the proposed annotations. By explicitly describing the 
relationships between multimedia resources, visual content and other support material and using Open LD datasets, the digital content from internal business repositories can be linked to each other. Other useful knowledge data and cross-application domain's visual resources and aids on the web, can also be linked.

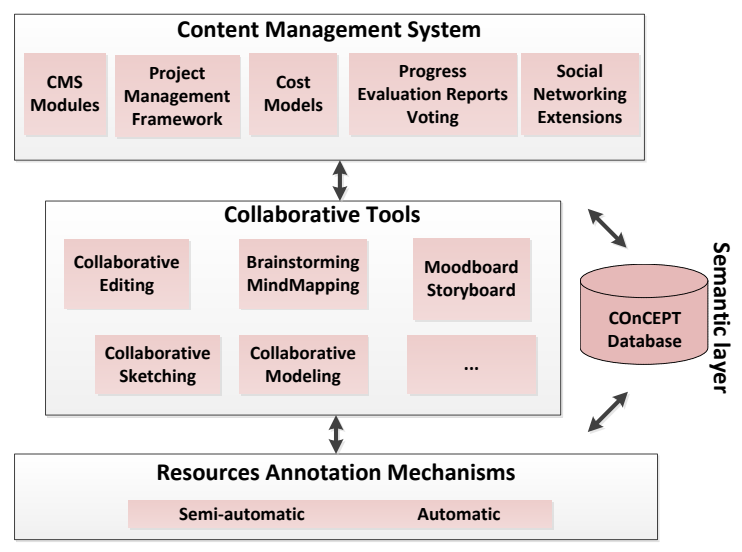

Figure 1: The COnCEPT platform framework

In addition to the core components of the COnCEPT architecture, a set of functionalities will be incorporated such as activities logging, roles and policies definition and management, online voting, cost model description and progress evaluation reports creation. Finally, a project management and social media framework will be implemented and integrated into the COnCEPT platform allowing for management of the projects by the participants. In addition it will also allow for the sharing of information with the client or social networks following the successful completion of crucial milestones within the design process.

\section{DESIGN FOR COLLABORATION}

Given the social nature of collaborative design, the project has approached the design activities of COnCEPT services by adopting an approach based on the conceptual framework Activity-based Theory (AT) (Kaptelinin \& Nardi, 1997). This approach allows us to frame human activity in relation to the people, activities, objects, tools and community involved. AT is specifically applicable to the COnCEPT platform as it analyses how tools mediate human activity and how these tools can be situated in a socio-cultural setting. User-Centred Design (UCD) research methods are being used to reveal the ways in which designers are working and to identify some of the opportunities for assisting the design process. For example keeping track of the development of the project when multiple parties are involved. This involves talking directly to professional designers and visiting their studios and making direct observations in order to record how they tackle new projects and collaborate with colleagues. In this section we introduce five design guidelines to promote collaboration, which has informed the design of the COnCEPT platform.

\subsection{Support for personalisation}

Design activities, as much as any other human activity, evolve constantly. COnCEPT should therefore allow its users to re-define and change their activities during the course of their work (Houben et al., 2013). Where customization takes into account the context of use, personalisation goes a step further by offering the ability of an interactive application capable of being adapted to suit personal preferences. Personalisation is essential to maximize usability for business information systems (Nielsen, 2011). In an integrated collaborative environment as targeted by COnCEPT, personalisation is envisioned on two levels. On one level, the collaborators will be able to use the appropriate tools depending on their expertise and design practices. On another level, personalisation will enable users to work more efficiently due to the adapted settings and parameters of these tools.

\subsection{Integrating collaborative tools}

The use of diverse standalone tools creates a context switch, which has been identified as a source of interruptions and project fragmentation, since most tools are disconnected from each other to perform similar or related activities (Houben et al., 2013). The COnCEPT platform aims to support both individual and team workflows, integrating tools based on these workflows in order to support more fluent collaboration. COnCEPT will constitute an activity-oriented workspace, where design activities are clearly integrated through the user interface (Houben et al., 2013) to support conceptual design activities in different individual and collaborative work settings.

\subsection{Supporting transparency and context- awareness}

Since design activities are not limited to a single context of use, in that they can be face-to-face, or in different times and locations, COnCEPT should 
ensure context-awareness among various devices and physical locations to accurately represent human activities. For example, sharing activities should not only be possible amongst users but also devices, and in consideration of these different contexts. This will be integrated in the COnCEPT platform making use of cloud storage functionalities in which files are transparently stored and made available online and on-demand instead of being located on a local server.

\subsection{Design as a social process}

Design activities are often interdisciplinary activities, and involve collaboration amongst customers, designers and technical specialists. Therefore, COnCEPT users should be able to know who is participating in the design process, and coordinate different activities in regards to these other participants. After determining the role of each discipline in the conceptual design phase, the COnCEPT platform will define an interaction protocol and devise a set of effective communication methods that aid the awareness and coordination of collaborative design activities.

In distributed collaboration, human-computer interaction must be closely coupled with humanhuman interaction and both types of interaction should not interfere with each other. Alternatively, the computer should discretely support, facilitate and enhance the human element of the process. The COnCEPT platform will investigate which interaction techniques are most suited for specific situations and activities for example individual versus collaborative activities, and how they can be combined into an integrated experience.

\subsection{Supporting awareness and reflection}

The approach adopted by COnCEPT, is to facilitate successful bonding and coordination between the team members through sharing information that helps individuals orient themselves to the stimuli provided and experienced by other team members (Shteynberg and Galinsky, 2011). This can be related to general attribution, i.e., according to the work of DeShon et al. (2004, p. 1037) "team-level constructs have their origins in individual cognition and behaviour and emerge as team members work together over time, in an interactive-task context". Facilitating feedback, and feed-forward, mechanisms as providers of information about the perception of other team members, can inspire selfreflection, as well as lead to implicit coordination of the whole team. This can be achieved by providing different perspectives on the design work inprogress, such as time-based, task-based, personbased, or activity-based views. Such perspectives contribute to a better understanding of the entire collaboration project, in that they might reveal links and hidden work patterns. Regularly changing perspectives can help designers and design stakeholders focus on the most relevant aspects of the collaboration and also may help to avoid motivational dips and conceptual cul-de-sacs. Selfreflection is considered to be useful in creativity when solving uncertain and undetermined problems (Schön, 1983), since it triggers divergent thinking, which is essential for identifying and properly representing the problem into list of actions to follow. Moreover, distributed design teams benefit from functional, inherent, and augmented information, which also provides coherent interactions between team members (Wensveen, et al., 2004).

Further research is needed on how to structure, collect, select, and present insightful information that triggers self-reflection at individual and team levels, and which supports the collaborative process as a whole. Considering that the backbone of intelligence is contextual knowledge, the boundary of knowledge management generalization is still yet to be identified that works for the individual, and which can be extended to other business domains.

\section{CONCLUSIONS}

The rapidly developing space of novel technologies that combine advances in knowledge management, context awareness and human machine interfaces may provide many new opportunities to facilitate the information exploitation needs of future business systems. This paper has presented the semantically driven user-centred COnCEPT approach, which is a step towards seamlessly integrating product development processes into a comprehensive context-aware collaborative design environment. The COnCEPT platform aims to mirror the professional design context and at the same time provide new and innovative tools to support the early stages of the design process by providing context-specific information and by supporting team- based collaboration. 


\section{ACKNOWLEDGEMENTS}

This research is funded by the European Commission 7th Framework ICT Research Programme. Further details can be found accessed at: http://www.concept-fp7.eu

\section{REFERENCES}

Aggarwal, J.K., \& RyooHuman, M.S. (2011). Activity Analysis: A Review. Journal ACM Computing Surveys, 43(3), 1-43

Arangarasan, R. and Gadh, R., (2000). Geometric modelling and collaborative design in a multi-modal multi-sensory virtual environment. In Proc. of ASME 2000 Design Engineering Technical Conferences and Computers and Information in Engineering Conference, 2000, pp. 10-13

Capgemini (2013), The new digital workplace Employee productivity, brand image, business value, Report, http://www.fr.capgemini.com/node/618601

DeShon, R. P., Kozlowski, S. W. J., Schmidt, A. M., Milner, K. R., \& Wiechmann, D. (2004). A multiplegoal, multilevel model of feedback effects on the regulation of individual and team performance. Journal of Applied Psychology, 89(6), 1035-1056.

Dey, A. K. (2001). Understanding and Using Context. Personal Ubiquitous Computing,5 (1), 4-7

Hong, J., Suh, E., \& Kim, S-J. (2009). Context-aware systems: A literature review and classification. Expert Systems with applications 36 (1), 8509-8522

Houben, S., Bardram, J. E., Vermeulen, J., Luyten, K., \& Coninx, K., (2013). Activity-Centric Support for Ad Hoc Knowledge Work - A Case Study of co-Activity Manager, In Proc. of the SIGCHI Conf. on Human factors in computing systems (CHI'13), April 27-May 2 2013, Paris, France, ACM press, New York, pp. 2263-2272.

Gartner, (2013), Gartners outlines 10 IT trends to watch, http://redmondmag.com/articles/2013/10/03/10-ittrends.aspx

Kaptelinin, V., and Nardi B. A., (1997) Activity theory: basic concepts and applications. In CHI '97 Extended Abstracts on Human Factors in Computing Systems (CHI EA '97), ACM press, New York, pp. 158-159.

Kan, H. Y., Duffy, V. G. and Su, C. J. (2001). An internet virtual reality collaborative environment for effective product design. Computers in Industry, 2001, 45, 197213.

Nielsen, J. (2011). Intranet Portals: Personalization Hot, Mobile Weak, Governance Essential, NN/g blog, web log post, 18 July, viewed 15 April 2014, http://www.nngroup.com/articles/intranet-portals/

Schön, D.A. (1983). The Reflective Practitioner: How Professionals Think in Action. Basic Books

Shteynberg, G., \& Galinsky, A. D. (2011). Implicit coordination: Sharing goals with similar others intensifies goal pursuit. Journal of Experimental Social Psychology, 47(6), 1291-1294.

Shyamsundar, N. and Gadh, R. (2001). Internet-based collaborative product design with assembly features and virtual design spaces. Computer Aided Design, 2001, 33, 637-651

Schmidt, A., (2012). Context-Aware Computing ContextAwareness, Context-Aware User Interfaces, and Implicit Interaction. Online education. Retrieved June 24, 2013 from http://www.interactiondesign.org/encyclopedia/contextaware_computing.htl

Xu, X. W., and Liu, T. (2003). A web-enabled PDM system in a collaborative design environment. Robotics and Computer-Integrated Manufacturing, 2003, 19(4), 315-328.

Yang, H. and Xue, D. (2003). Recent research on developing Web-based manufacturing systems: a review. International Journal of Product Research, 2003, 41(15), 3601-3629.

Wensveen, S. A. G., Djajadiningrat, J. P., and Overbeeke, C. J. (2004). Interaction frogger: a design framework to couple action and function through feedback and feedforward. In Proceedings of the 5th conference on Designing interactive systems: processes, practices, methods, and techniques (DIS '04). ACM, New York, NY, USA, 177-184.

Wiki (2014). List of collaborative software, http://en.wikipedia.org/wiki/List_of_collaborative_sof tware 